\title{
Proctitis Caused by Mycobacterium avium-intracellulare in an HIV-Infected Patient
}

\author{
Jose Armando Gonzales Zamora ${ }^{1, *(1)}$ and Clara Milikowski ${ }^{2}$ \\ 1 Division of Infectious Diseases, Department of Medicine, Miller School of Medicine, University of Miami, \\ Miami, FL 33136, USA \\ 2 Division of Anatomic Pathology, Department of Pathology and Laboratory Medicine, University of Miami, \\ Miami, FL 33136, USA; CMilikowski@med.miami.edu \\ * Correspondence: jxg1416@med.miami.edu or jgonzales2010@hotmail.com; Tel.: +1-706-284-3510; \\ Fax: +1-305-243-4037
}

Received: 20 April 2018; Accepted: 5 May 2018; Published: 8 May 2018

\begin{abstract}
Infectious proctitis is usually associated with sexually transmitted diseases, especially in HIV-infected individuals. Limited information is found about the role of Mycobacterium avium-intracellulare as a causative agent for this condition. Here, we report the case of an HIV-infected patient with a CD4 count of 304 cells/uL and undetectable HIV viral load, who presented with constipation and painful defecation. Endoscopic evaluation was significant for shallow rectal ulcerations. Histopathology revealed poorly formed granulomas. Stool culture grew Mycobacterium sp. that was further identified as Mycobacterium avium-intracellulare by DNA probe. He was successfully treated with a 3-drug regimen that included azithromycin, ethambutol and rifabutin. We advocate the use of AFB stool culture in cases of proctitis in which initial investigations for sexually transmitted diseases are unrevealing.
\end{abstract}

Keywords: proctitis; Mycobacterium avium-intracellulare; HIV

\section{Introduction}

Proctitis is an inflammation of the rectal mucosa, and is frequently encountered in HIV-infected men who have sex with men (MSM). In this population, sexually transmitted diseases constitute the leading cause of infectious proctitis. The most common etiologic agents are Herpes simplex, Chlamydia trachomatis and Neisseria gonorrhoeae among others; however, a specific cause cannot be identified in a high number of cases [1]. Mycobacterium avium-intracellulare (MAI) is an environmental pathogen that has not been recognized as a causative agent of proctitis. This non-tuberculous Mycobacterium is commonly responsible for disseminated disease in severely immunocompromised HIV patients with CD4 counts lower than 50 cells/uL. Gastrointestinal involvement has been documented in several reports, with the duodenum, being the most commonly affected organ [2]. Here, we describe the case of an HIV-infected patient with localized proctitis secondary to Mycobacterium avium-intracellulare that was successfully treated with a three-drug regimen. Rectal involvement by MAI has been reported only as part of a disseminated process affecting the colon or other extraintestinal organs. To the best of our knowledge, the present case is the first description of MAI infection exclusively affecting the rectum. In this report, we also discuss the challenges we usually face for the diagnosis and management of this infection.

\section{Case Report}

A 48-year-old man originally from Mexico presented to the Infectious Disease clinic with constipation and painful defecation in the last 4 weeks. He had noticed streaks of blood on 
the toilet paper after wiping. He had a 5-year history of HIV infection treated initially with Tenofovir/emtricitabine/efavirenz that was recently switched to abacavir/lamivudine/dolutegravir. His latest CD4 count was 304 cells / uL and his HIV viral load was undetectable. He was MSM (men who have sex with men), but denied unprotected sex in the last 3 months. His vital signs were within normal limits. He weighed $77.1 \mathrm{~kg}$ and did not report any significant weight loss. His abdomen was soft, nontender and non-distended. Bowel sounds were normoactive. Rectal exam was normal. Laboratory studies showed hemoglobin of $14.1 \mathrm{~g} / \mathrm{dL}$, leukocyte count of $4.7 \mathrm{~K}$ cells $/ \mathrm{uL}$ and platelet count of $246 \mathrm{~K}$ cells / uL. Chemistry panel was significant only for mildly elevated alkaline phosphatase (142 U/L). RPR (rapid plasma reagin) was negative. Stool culture was negative for Salmonella, Shigella, Aeromona and Plesiomona. Campylobacter antigen and Escherichia coli shigatoxins were not detected. Ova and parasites were not isolated in stool studies. He underwent rectal swab for gonorrhea and chlamydia PCR, which gave negative results. Given unrevealing work-up, the patient was referred to Gastroenterology for endoscopic evaluation. Colonoscopy showed moderate inflammation characterized by congestion, erythema and friability of the rectal mucosa. Shallow ulcerations were noted only in the rectum (Figure 1). The rest of the colon did not show any abnormalities. Histopathology examination of rectal tissue disclosed a mucosa with increased lymphoplasmacytic and neutrophilic infiltrate in lamina propia. Acute cryptitis and focal crypt abscesses were noted, along with erosions and few poorly formed granulomas (Figure 2). Immunohistochemistry for HSV1, HSV2, and CMV was negative. Tissue AFB (acid-fast bacilli) staining did not show any organisms. AFB smear in stool was negative; however, two weeks later, stool culture grew Mycobacterium sp. that was further identified as Mycobacterium avium-intracellulare complex by DNA probe. No susceptibility testing was performed. Rectal tissue culture did not grow any organisms. To complete the work-up, a chest $X$-ray and quantiferon gold were ordered. The results were unremarkable. The patient was started on azithromycin $500 \mathrm{mg}$ daily, rifabutin $300 \mathrm{mg}$ daily and ethambutol $1200 \mathrm{mg}$ daily. At 2-month follow up, the patient reported complete resolution of his symptoms. Four months later, rifabutin was discontinued. The plan was to continue with azithromycin and ethambutol for six additional months to complete a total treatment course of one year.

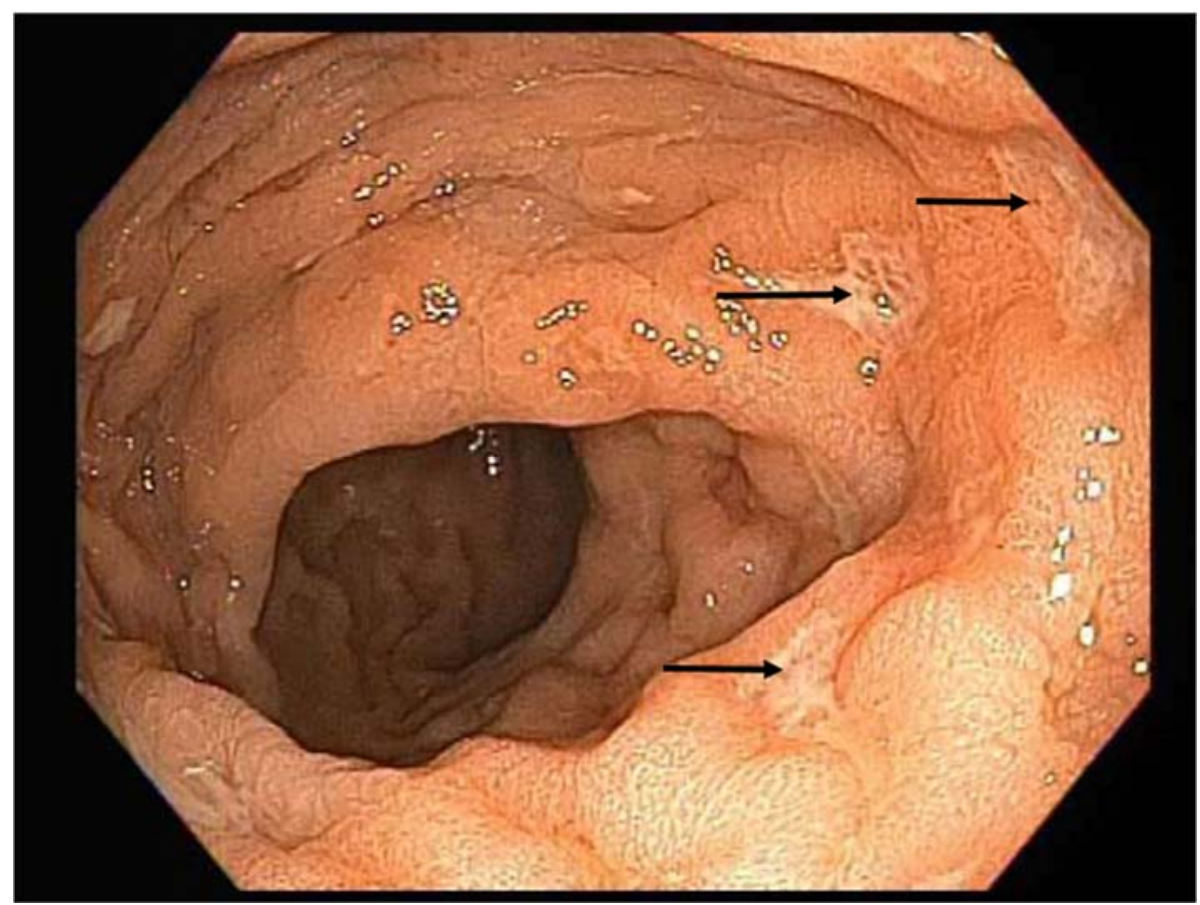

Figure 1. Colonoscopy showing shallow ulcerations in rectal mucosa (arrow). 


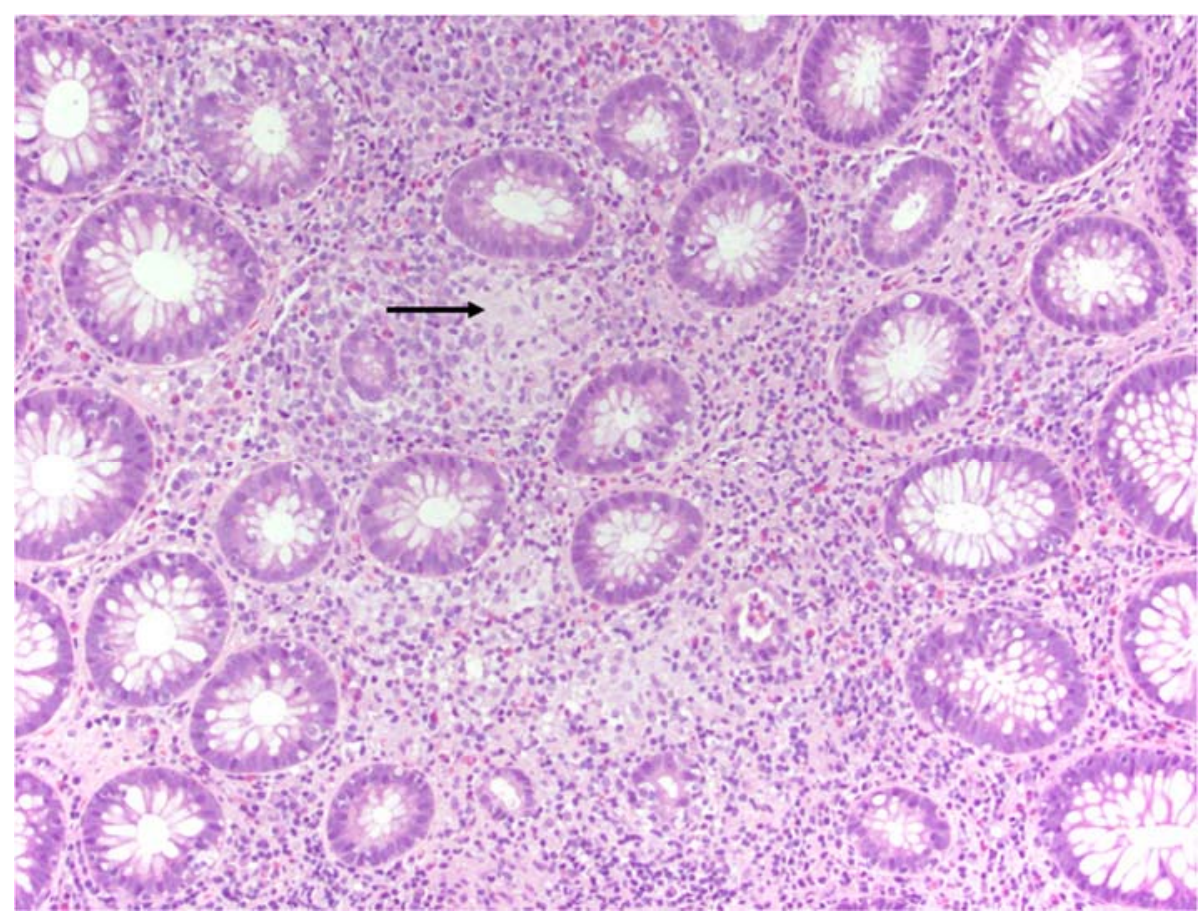

Figure 2. Histopathology showing cryptitis and noncaseating granuloma (arrow) in rectal mucosa $(\mathrm{H} \& \mathrm{E}, 400 \times)$.

\section{Discussion}

Proctitis is an inflammation of the rectal mucosa and is restricted to the distal $15 \mathrm{~cm}$ of the colon. Symptoms vary depending on the specific pathological process. The most common symptom is a continuous urge to have a bowel movement. Other symptoms include anorectal pain, anal discharge, constipation and rectal bleeding [3]. This condition has several non-infectious and infectious causes. In cases of infectious proctitis, the cause is usually a sexually transmitted disease. In a retrospective study conducted by Klausner et al. in San Francisco, the most common pathogens were gonorrhea $(30 \%)$, chlamydia $(19 \%), \mathrm{HSV}-2(16 \%)$ and syphilis $(2 \%)$. No identifiable infectious source was found in $46 \%$ of cases. Co-infections were not uncommon, $10 \%$ of the patients with infectious proctitis tested positive for multiple pathogens [4].

The HIV status is a factor that seems to influence the etiological distribution of pathogens associated with proctitis. Bissessor et al. conducted a study in Australia that aimed to compare the spectrum of microorganisms responsible for infectious proctitis between HIV-infected and HIV-non-infected men who have sex with men. They found that, compared with the rest of the study cohort, HIV-infected individuals had a higher rate of proctitis caused by lymphogranuloma venereum ( $7.8 \%$ vs. $0.7 \%, p=0.004)$, HSV-1 ( $14.2 \%$ vs. $6.5 \%, p=0.04), \mathrm{HSV}-2(22 \%$ vs. $12.3 \%, p=0.03)$ and multiple infections ( $17.7 \%$ vs. $8.6 \%, p=0.017$ ). Of note, $28 \%$ of HIV-infected individuals did not have any pathogen detected, which was significantly lower that the percentage observed in HIV-negative patients [5].

Our patient presented classic symptoms of proctitis; however, appropriate investigations for sexually transmitted infections were unrevealing. Further work-up disclosed the diagnosis of MAI proctitis. This non-tuberculous Mycobacterium has been recognized as one of the most common etiologies of opportunistic infections in patients with acquired immunodeficiency syndrome (AIDS). MAI is ubiquitous in the environment and the mode of infection seems to be through inhalation or ingestion. In homosexual men, venereal transmission has also been suggested [6]. In our patient, there was no evidence of pulmonary involvement and he denied any recent sexual contact. We believe that rectal seeding may have occurred via hematogenous spread or by ingestion. 
The risk of MAI infection in patients with HIV increases as the CD4 count drops below 50 cells/uL. One of the unique characteristics of our patient is his relatively high CD4 count ( 304 cells/uL) on presentation, which is uncommon in HIV patients with MAI infection. There may be a genetic predisposition for acquiring this infection. In a case-control study from the Multicenter AIDS Cohort, specific human leukocyte antigen (HLA) class II alleles (DRB1, DQB, DM) were more commonly associated with MAI disease [7]. An innate susceptibility could explain the development of MAI infection in our patient.

The two main manifestations of MAI infection in HIV-infected patients are disseminated and localized disease. Disseminated disease was the predominant form prior to the widespread use of antiretroviral therapy. The symptoms of disseminated MAI are nonspecific and include fever, night sweats, diarrhea and abdominal pain. Gastrointestinal symptoms are reported in approximately $40 \%$ of disseminated cases and they include diarrhea, malabsorption and gastrointestinal hemorrhage [8-10]. Within the gastrointestinal tract, the duodenum is the most commonly involved organ. The case series published by Gray et al. describes duodenal involvement in $88 \%$ of cases of gastrointestinal MAI infection. Other affected organs were the esophagus and the liver. Rectal involvement has also been described, but as part of a disseminated process affecting the colon and other extra-intestinal areas $[2,11]$. In our patient, only the rectal mucosa was involved, which constitutes a presentation never reported before.

The diagnosis of MAI infection typically relies on cultures. In cases of disseminated infection, blood culture is the initial preferred diagnostic test, given its high sensitivity and lower invasiveness. In this scenario, there is a limited role for obtaining stool cultures. A prospective study found that colonization of the gastrointestinal and respiratory tract had a sensitivity of only $20 \%$ and a positive predictive value of $60 \%$ for detecting disseminated disease [12]. On the other hand, in cases of proven gastrointestinal involvement, stool culture has demonstrated to be a valuable test, with an overall sensitivity as high as $86 \%$ and a specificity of $99 \%[2,13,14]$. Tissue culture has shown lower detection rates $(76 \%)$. The histopathology of MAI infection is usually characterized by noncaseating granulomas resembling Crohn's disease. Small bowel biopsies can also show severe villous flattening with aggregates of foamy macrophages full of intracellular acid-fast organisms $[15,16]$. One of the distinctive endoscopic features noted in cases of duodenal MAI infection is the presence of diffusely whitish nodules, found in approximately $35 \%$ of cases [2,17]. Mucosal friability, superficial ulceration and diffuse erythema are endoscopic characteristics described in cases of MAI colitis [10]. In our patient, the diagnosis was achieved by stool culture and histology; and it was subsequently confirmed by adequate response to anti-mycobacterial treatment.

In patients with AIDS, combination antimicrobial therapy is recommended for treatment of MAI infection. Dual therapy with macrolides and ethambutol is the cornerstone of MAI treatment. A third agent is usually added in cases of advanced immunosuppression, high mycobacterial loads, or absence of effective antiretroviral therapy [18]. Rifabutin is the preferred third drug, but other alternatives include levofloxacin, moxifloxacin or amikacin. Treatment should be continued for at least 12 months; however, the ultimate duration depends upon how fast the patient recovers his immunologic function after initiating antiretroviral therapy, since the CD4 count should be stably above 100 cells/uL for at least six months before discontinuing treatment [18]. In HIV-non-infected patients, the duration of therapy depends on the organ involved. In cases of pulmonary MAI infection, the treatment should be continued until sputum cultures are consecutively negative for 12 months. Since sputum conversion usually takes 3 to 6 months of treatment, a typical patient is treated for 15 to 18 months. In cases of extrapulmonary MAI infection, the optimal duration of treatment has not been well established, and treatment is habitually administered for at least 6 months [19]. We decided to treat our patient for 1 year given his immunocompromised state; however, he was not in late stages of HIV infection, as are most of the patients included in studies of disseminated MAI. This could raise the point that a shorter course of treatment could have probably been equally effective in our patient. Further studies 
are needed to determine the optimal duration of treatment in cases of localized extrapulmonary MAI infection, especially when CD4 counts are above 200 cells/uL.

\section{Conclusions}

Proctitis secondary to MAI is very uncommon, even in the setting of HIV infection. We believe that stool AFB and culture should be part of the work-up in patients presenting with proctitis, in whom initial investigations for sexually transmitted diseases are negative. The treatment for MAI is typically based on a 3-drug regimen that includes macrolides, ethambutol and possibly rifabutin. The optimal duration of therapy for extrapulmonary disease has not been standardized; however, a six- to twelve-month course is usually advocated.

Consent for Publication: Written informed consent was obtained from the patient for publication of this case report and any supplementary images.

Conflicts of Interest: The authors declare no conflict of interest.

\section{References}

1. Rompalo, A.M. Diagnosis and treatment of sexually acquired proctitis and proctocolitis: An update. Clin. Infect. Dis. 1999, 28, S84-S90. [CrossRef] [PubMed]

2. Gray, J.R.; Rabeneck, L. Atypical mycobacterial infection of the gastrointestinal tract in AIDS patients. Am. J. Gastroenterol. 1989, 84, 1521-1524. [PubMed]

3. Hamlyn, E.; Taylor, C. Sexually transmitted proctitis. Postgrad. Med. J. 2006, 82, 733-736. [CrossRef] [PubMed]

4. Klausner, J.D.; Kohn, R.; Kent, C. Etiology of clinical proctitis among men who have sex with men. Clin. Infect. Dis. 2004, 38, 300-302. [CrossRef] [PubMed]

5. Bissessor, M.; Fairley, C.K.; Read, T.; Denham, I.; Bradshaw, C.; Chen, M. The etiology of infectious proctitis in men who have sex with men differs according to HIV status. Sex. Transm. Dis. 2013, 40, 768-770. [CrossRef] [PubMed]

6. Damsker, B.; Bottone, E.J. Mycobacterium avium-mycobacterium intracellulare from the intestinal tracts of patients with the acquired immunodeficiency syndrome: Concepts regarding acquisition and pathogenesis. J. Infect. Dis. 1985, 151, 179-181. [CrossRef] [PubMed]

7. Naik, E.; LeBlanc, S.; Tang, J.; Jacobson, L.P.; Kaslow, R.A. The complexity of HLA class II (DRB1, DQB1, DM) associations with disseminated Mycobacterium avium complex infection among HIV-1-seropositive whites. J. Acquir. Immune Defic. Syndr. 2003, 33, 140-145. [CrossRef] [PubMed]

8. Zakowski, P.; Fligiel, S.; Berlin, G.W.; Johnson, L., Jr. Disseminated Mycobacterium avium-intracellulare infection in homosexual men dying of acquired immunodeficiency. JAMA 1982, 248, 2980-2982. [CrossRef] [PubMed]

9. Sohn, C.C.; Schroff, R.W.; Kliewer, K.E.; Lebel, D.M.; Fligiel, S. Disseminated Mycobacterium avium-intracellulare infection in homosexual men with acquired cell-mediated immunodeficiency: A histologic and immunologic study of two cases. Am. J. Clin. Pathol. 1983, 79, 247-252. [CrossRef] [PubMed]

10. Keller, C.; Kirkpatrick, S.; Lee, K.; Paul, M.; Hanson, I.C.; Gilger, M. Disseminated Mycobacterium avium complex presenting as hematochezia in an infant with rapidly progressive acquired immunodeficiency syndrome. Pediatr. Infect. Dis. J. 1996, 15, 713-715. [CrossRef] [PubMed]

11. Wolke, A.; Meyers, S.; Adelsberg, B.R.; Bottone, E.J.; Damsker, B.; Schwartz, I.S.; Janowitz, H.D. Mycobacterium avium-intracellulare-associated colitis in a patient with the acquired immunodeficiency syndrome. J. Clin. Gastroenterol. 1984, 6, 225-229. [PubMed]

12. Chin, D.P.; Hopewell, P.C.; Yajko, D.M.; Vittinghoff, E.; Horsburgh, C.R., Jr.; Hadley, W.K.; Stone, E.N.; Nassos, P.S.; Ostroff, S.M.; Jacobson, M.A. Mycobacterium avium complex in the respiratory or gastrointestinal tract and the risk of M. avium complex bacteremia in patients with human immunodeficiency virus infection. J. Infect. Dis. 1994, 169, 289. [CrossRef] [PubMed]

13. Hellyer, T.J.; Brown, I.N.; Taylor, M.B.; Allen, B.W.; Easmon, C.S. Gastro-intestinal involvement in Mycobacterium avium-intracellulare infection of patients with HIV. J. Infect. 1993, 26, 55-66. [CrossRef] 
14. Morris, A.; Reller, L.B.; Salfinger, M.; Jackson, K.; Sievers, A.; Dwyer, B. Mycobacteria in stool specimens: The nonvalue of smears for predicting culture results. J. Clin. Microbiol. 1993, 31, 1385-1387. [PubMed]

15. Ishikane, M.; Tanuma, J. Mycobacterium avium complex enteritis in HIV-infected patient. IDCases 2014, 1, 22-23. [CrossRef] [PubMed]

16. Nguyen, H.N.; Frank, D.; Handt, S.; Rieband, H.C.; Maurin, N.; Sieberth, H.G.; Matern, S. Severe gastrointestinal hemorrhage due to Mycobacterium avium complex in a patient receiving immunosuppressive therapy. Am. J. Gastroenterol. 1999, 94, 232-235. [CrossRef] [PubMed]

17. Monsour, H.P., Jr.; Quigley, E.M.; Markin, R.S.; Dalke, D.D.; Goldsmith, J.C.; Harty, R.F. Endoscopy in the diagnosis of gastrointestinal Mycobacterium avium-intracellulare infection. J. Clin. Gastroenterol. 1991, 13, 20-24. [CrossRef] [PubMed]

18. Panel on Opportunistic Infections in HIV-Infected Adults and Adolescents. Guidelines for the Prevention and Treatment of Opportunistic Infections in HIV-Infected Adults and Adolescents: Recommendations from the Centers for Disease Control and Prevention, the National Institutes of Health, and the HIV Medicine Association of the Infectious Diseases Society of America. Available online: http://aidsinfo.nih.gov/ contentfiles/lvguidelines/adult_oi.pdf (accessed on 20 April 2018).

19. Griffith, D.E.; Aksamit, T.; Brown-Elliott, B.A.; Catanzaro, A.; Daley, C.; Gordin, F.; Holland, S.M.; Horsburgh, R.; Huitt, G.; Iademarco, M.F.; et al. An official ATS/IDSA statement: Diagnosis, treatment, and prevention of nontuberculous mycobacterial diseases. Am. J. Respir. Crit. Care Med. 2007, 175, 367-416. [CrossRef] [PubMed]

(C) 2018 by the authors. Licensee MDPI, Basel, Switzerland. This article is an open access article distributed under the terms and conditions of the Creative Commons Attribution (CC BY) license (http:/ / creativecommons.org/licenses/by/4.0/). 